\title{
Comparison Study on the Exact Dynamic Stiffness Method for Free Vibration of Thin and Moderately Thick Circular Cylindrical Shells
}

\author{
Xudong Chen ${ }^{1}$ and Kangsheng $\mathrm{Ye}^{2}$ \\ ${ }^{1}$ School of Naval Architecture and Civil Engineering, Jiangsu University of Science and Technology, Zhangjiagang 215600, China \\ ${ }^{2}$ Department of Civil Engineering, Tsinghua University, Beijing 100084, China \\ Correspondence should be addressed to Kangsheng Ye; yeks@tsinghua.edu.cn
}

Received 3 September 2016; Revised 21 November 2016; Accepted 24 November 2016

Academic Editor: Marcello Vanali

Copyright ( $) 2016$ X. Chen and K. Ye. This is an open access article distributed under the Creative Commons Attribution License, which permits unrestricted use, distribution, and reproduction in any medium, provided the original work is properly cited.

Comparison study on free vibration of circular cylindrical shells between thin and moderately thick shell theories when using the exact dynamic stiffness method (DSM) formulation is presented. Firstly, both the thin and moderately thick dynamic stiffness formulations are examined. Based on the strain and kinetic energy, the vibration governing equations are expressed in the Hamilton form for both thin and moderately thick circular cylindrical shells. The dynamic stiffness is assembled in a similar way as that in classic skeletal theory. With the employment of the Wittrick-Williams algorithm, natural frequencies of circular cylindrical shells can be obtained. A FORTRAN code is written and used to compute the modal characteristics. Numerical examples are presented, verifying the proposed computational framework. Since the DSM is an exact approach, the advantages of high accuracy, no-missing frequencies, and good adaptability to various geometries and boundary conditions are demonstrated. Comprehensive parametric studies on the thickness to radius ratio $(h / r)$ and the length to radius ratio $(L / r)$ are performed. Applicable ranges of $h / r$ are found for both thin and moderately thick DSM formulations, and influences of $L / r$ on frequencies are also investigated. The following conclusions are reached: frequencies of moderately thick shells can be considered as alternatives to those of thin shells with high accuracy where $h / r$ is small and $L / r$ is large, without any observation of shear locking.

\section{Introduction}

Free vibration analysis of shell structures is important for further dynamic analysis in engineering. Among all shell types, circular cylindrical shells are the most widely used in a good variety of applications, for example, tubes, containment vessels, and aircraft fuselages. Therefore, exact modal characteristic analysis of circular cylindrical shells is of considerable scientific and engineering significance.

It is well known that shell theories can be categorised into thin, moderately thick, and thick forms. Since the KirchhoffLove hypothesis for thin elastic shell theory was established [1], much effort was spent on its vibration development. Arnold and Warburton [2] analysed the flexural vibration of thin cylindrical shells with general boundary conditions using Rayleigh's principle. Leissa [3] provided a systematic review and summary on the vibration of different shell theories and shell types. Bardell et al. [4] investigated the free vibration of isotropic open cylindrical shell panels by applying an $h-p$ version finite element method. Tan [5] introduced a substructuring method for predicting the natural frequencies of circular cylindrical shells with arbitrary boundary conditions.

On the other hand, moderately thick shell theory which derives from the Reissner-Mindlin hypothesis is less frequently used in analysing the free vibration behaviour of shells due to its complexity. Naghdi and Cooper [6] conducted vibration analysis on circular cylindrical shells with the consideration of shear deformation and rotary inertia by employing elastic wave method. Mirsky and Herrmann [7] studied the nonaxisymmetric vibration of moderately thick circular cylindrical shells. Tornabene and Viola [8] proposed 
an approach to analyse free vibration of parabolic shells using Generalised Differential Quadrature method (GDQ) based on the first-order shear deformation theory. Mantari et al. [9] applied a new accurate higher-order shear deformation theory to perform free vibration analysis of multilayered shells.

Although the free vibration of shells has been extensively examined, there are continuing research efforts made with new methods and shell types, for example, $p$-version mixed finite element by Kim et al. [10]; GDQ method for doubly curved shells of revolution by Tornabene et al. [11, 12]; higherorder theory for thin-walled beams by Pagani et al. [13]; and three-dimensional analysis method by Ye et al. $[14,15]$.

The dynamic stiffness method (DSM) is an exact method since the dynamic stiffness is computed directly from the exact vibration governing equations. It is suitable for computational analysis of free vibration of continuous systems with infinite degrees of freedom. In conjunction with the Wittrick-Williams (W-W) algorithm $[16,17]$ and the recursive Newton's method $[18,19]$, the DSM has been employed in free vibration of skeletal structures, for example, nonuniform Timoshenko beams [20], three-layered sandwich beams [21], and functionally graded beams [22]. Pagani et al. [23] employed the exact dynamic stiffness elements to investigate the free vibration of thin-walled structures. A recent paper [24] applied the DSM to the free vibration analysis of thin circular cylindrical shells. The present authors used to employ the DSM to investigate the free vibration of shells of revolution [25], and the complete theory was given in [26] based on the thin shell assumption. Further extension of the DSM to moderately thick circular cylindrical shells [27] was made with the consideration of transverse shear deformation and rotary inertia.

As an exact method, the dynamic stiffness method has the following advantages:

(1) The method is exact and theoretically solutions can be obtained with any desired accuracy.

(2) The original eigenvalue problems of two-dimensional partial differential equations (PDEs) are degraded to the eigenvalue problems of a set of one-dimensional ordinary differential equations (ODEs) by introducing the circumferential vibration modes. Since the method is exact, frequencies and vibration modes of any order can be calculated with a small number of elements.

(3) The Wittrick-William algorithm is mathematically strict and ensures that no eigenvalue will be omitted.

(4) The DSM formulation is general and versatile, making it capable of solving the free vibration of circular cylindrical shells with a variety of geometries and arbitrary boundary conditions.

Though the free vibration formulation using the DSM has been established for both thin and moderately thick circular cylindrical shells, there is no comprehensive comparison study available currently to address the applicability of DSM formulations between the two different shell theories. This paper attempts to show the compatibility on the free vibration

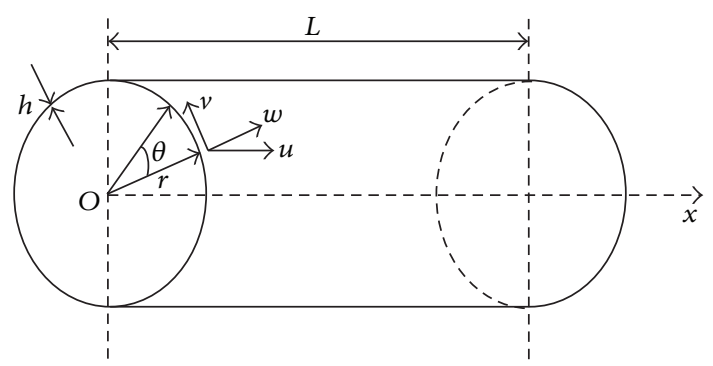

FIGURE 1: Coordinates of a circular cylindrical shell.

of circular cylindrical shells between thin and moderately thick DSM formulations. The DSM formulations of both thin and moderately thick circular cylindrical shells are well compared in Sections 2 and 3. Afterwards, the Wittrick-Williams algorithm is presented, providing a solution to computing the number of clamped-end natural frequencies $J_{0}$. Four numerical examples are given in Section 5, demonstrating the capability and reliability of the proposed method. Extensive parametric studies are performed in Section 6 on the ratios of thickness to radius $(h / r)$ and length to radius $(L / r)$ to find out the applicable ranges of each theory. Differences between frequencies from thin and moderately thick DSM formulations are analysed. The following conclusions are reached in the final: the compatibility of DSM between thin and moderately thick formulations is proven without shear locking.

\section{Vibration Equations}

This section theoretically presents a comparison study on DSM formulations for free vibration of both thin and moderately thick circular cylindrical shells. Figure 1 shows the coordinate system of a homogeneous, isotropic, and circumferentially closed circular cylindrical shell. The axial and circumferential directions of the shell are termed as $x$ and $\theta$, respectively. The length of the shell is $L$, radius is $r$, and the thickness is $h$. Young's modulus is $E$, Poisson's ratio is $v$, and the density is $\rho$.

For thin shells, the vibration can be represented by the translational displacements of the middle surface $\{\Delta\}_{\text {thin }}=$ $\{u, v, w\}^{\mathrm{T}}$ along $x, \theta$, and $r$ directions, while, for moderately thick shells, the angular displacements $\left\{\psi_{x}, \psi_{\theta}\right\}^{\mathrm{T}}$ of the middle surface along $x$ and $\theta$ directions are independent, which results in an expansion of the displacement vector to $\{\boldsymbol{\Delta}\}_{\text {thick }}=\left\{u, v, w, \psi_{x}, \psi_{\theta}\right\}^{\mathrm{T}}$.

The strain-displacement relationship at the middle surface of a circular cylindrical shell can be written in the form of

$$
\{\boldsymbol{\varepsilon}\}=[\mathbf{L}]\{\boldsymbol{\Delta}\},
$$

where $\{\boldsymbol{\varepsilon}\}$ is the strain vector and $\{\Delta\}$ is the displacement vector which can be $\{\boldsymbol{\Delta}\}_{\text {thin }}$ or $\{\boldsymbol{\Delta}\}_{\text {thick }}$ as mentioned above. The expressions of the differential matrix $[\mathbf{L}]$ for both thin and moderately thick circular cylindrical shells can be found in [3]. 


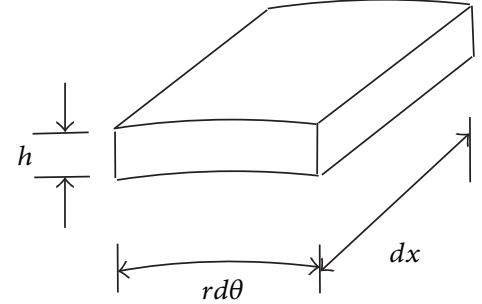

FIgURE 2: The diagram of an infinitesimal portion of a circular cylindrical shell.

Similarly, the internal force-strain relationship at the middle surface of a circular cylindrical shell can also be expressed as

$$
\{\mathbf{N}\}=[\mathbf{D}]\{\boldsymbol{\varepsilon}\},
$$

where $\{\mathbf{N}\}$ is the internal force vector and $[\mathbf{D}]$ is the stiffness matrix which can also be found in [3].

Denote $d A$ as the area of an infinitesimal portion in the middle surface of a circular cylindrical shell (see Figure 2), and the area of this infinitesimal portion is $d A=r d \theta d x$. The total strain energy $U$ for free vibration of the circular cylindrical shell can be integrated along the whole middle surface $S$ as

$$
U=\iint_{S} \frac{1}{2}\{\boldsymbol{\varepsilon}\}^{\mathrm{T}}[\mathbf{D}]\{\boldsymbol{\varepsilon}\} d A
$$

and the total free vibration kinetic energy is obtained as

$$
T=\frac{\rho}{2} \iint_{S} h\left[\left(\frac{\partial u}{\partial t}\right)^{2}+\left(\frac{\partial v}{\partial t}\right)^{2}+\left(\frac{\partial w}{\partial t}\right)^{2}\right] d A
$$

for thin shell, or

$$
\begin{aligned}
T & =\frac{\rho}{2} \iint_{S}\left\{h\left[\left(\frac{\partial u}{\partial t}\right)^{2}+\left(\frac{\partial v}{\partial t}\right)^{2}+\left(\frac{\partial w}{\partial t}\right)^{2}\right]\right. \\
& \left.+\frac{h^{3}}{12}\left[\left(\frac{\partial \psi_{x}}{\partial t}\right)^{2}+\left(\frac{\partial \psi_{\theta}}{\partial t}\right)^{2}\right]\right\} d A
\end{aligned}
$$

for moderately thick circular cylindrical shells.

The dynamic displacement functions $\{\boldsymbol{\Delta}\}_{\text {thin }}$ and $\{\boldsymbol{\Delta}\}_{\text {thick }}$ are expressed differently as

$$
\begin{aligned}
\{\boldsymbol{\Delta}\}_{\text {thin }}= & \left\{\begin{array}{c}
u_{n}(x) \cos n \theta \sin \omega t \\
v_{n}(x) \sin n \theta \sin \omega t \\
w_{n}(x) \cos n \theta \sin \omega t
\end{array}\right\}, \\
\{\boldsymbol{\Delta}\}_{\text {thick }}= & \left\{\begin{array}{c}
u=u_{n}(x) \cos n \theta \sin \omega t \\
v=v_{n}(x) \sin n \theta \sin \omega t \\
w=w_{n}(x) \cos n \theta \sin \omega t \\
\psi_{x}=\psi_{x n}(x) \cos n \theta \sin \omega t \\
\psi_{\theta}=\psi_{\theta n}(x) \sin n \theta \sin \omega t
\end{array}\right\} .
\end{aligned}
$$

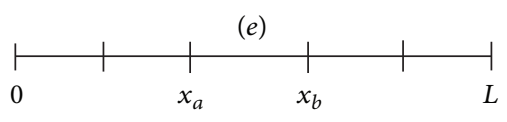

FIgURE 3: The mesh division along the axis.

$n$ is the circumferential wave number; $\omega$ is a circular frequency; $u_{n}(x), v_{n}(x), w_{n}(x), \psi_{x n}(x)$, and $\psi_{\theta n}(x)$ are axial vibration functions.

The Hamilton principle suggests

$$
\delta \int_{t_{1}}^{t_{2}}(U-T) d t=0
$$

where $\left(t_{1}, t_{2}\right)$ is an arbitrary time interval during vibration. The equations of motion can be derived from (7). Substituting the dynamic displacement functions (6) into equations of motion, the free vibration governing differential equations can be obtained. By rewriting the governing differential equations into the Hamilton form, generalised displacements $\{\mathbf{u}\}$ and forces $\{\mathbf{v}\}$ are obtained, where $\{\mathbf{u}\}$ and $\{\mathbf{v}\}$ are given in the Appendix.

The vibration governing equations can be written in the Hamilton form of

$$
[\mathbf{J}]\left\{\mathbf{z}^{\prime}\right\}=[\mathbf{S}]\{\mathbf{z}\},
$$

where ()$^{\prime}$ denotes the differentiation with respect to $x$ and $\{\mathbf{z}\}=\left\{\begin{array}{ll}\mathbf{u} & \mathbf{v}\end{array}\right\}^{\mathrm{T}}$. [J] $=\left[\begin{array}{cc}0 & -\mathbf{I} \\ \mathbf{I} & \mathbf{0}\end{array}\right]$ and $\mathbf{I}$ is an identity matrix with the order of four (or five) for thin (or moderately thick) shells. The coefficient matrix $[\mathbf{S}]$ is a symmetric eighth- (or tenth-) order matrix for thin (or moderately thick) circular cylindrical shells. Details of $[\mathbf{S}]$ can also be found in the Appendix.

\section{Dynamic Stiffness Matrix}

Should the circumferential wave number $n$ be a specific value, (8) is one-dimensional with respect to $x$ only. Therefore, a circular cylindrical shell can be analysed as a general one-dimensional skeleton-like structure with four (or five) degrees of freedom $\left\{\begin{array}{lllll}u_{1} & u_{2} & u_{3} & u_{4}\end{array}\right\}$ (or $\left\{\begin{array}{lllll}u_{1} & u_{2} & u_{3} & u_{4} & u_{5}\end{array}\right\}$ ) for thin (or moderately thick) shells. Accordingly, the internal force vectors for both thin and moderately thick shell theories are $\left\{\begin{array}{llll}v_{1} & v_{2} & v_{3} & v_{4}\end{array}\right\}^{\mathrm{T}}$ and $\left\{\begin{array}{lllll}v_{1} & v_{2} & v_{3} & v_{4} & v_{5}\end{array}\right\}^{\mathrm{T}}$, respectively. The dynamic stiffness of free vibration of a circular cylindrical shell can now be set up in a similar way as that in classic onedimensional skeletal theory.

Figure 3 shows that a circular cylinder is divided into several shell segments along its axial direction. Taking element (e), for example, the $x$ coordinates at its two ends are $x_{a}$ and 
$x_{b}$, respectively. The segment-end displacement vector $\{\mathbf{d}\}^{e}$ is defined as

$$
\begin{gathered}
\{\mathbf{d}\}_{\text {thin }}^{e}=\left\{u_{1}\left(x_{a}\right), u_{2}\left(x_{a}\right), u_{3}\left(x_{a}\right), u_{4}\left(x_{a}\right), u_{1}\left(x_{b}\right),\right. \\
\left.u_{2}\left(x_{b}\right), u_{3}\left(x_{b}\right), u_{4}\left(x_{b}\right)\right\}^{\mathrm{T}}, \\
\{\mathbf{d}\}_{\text {thick }}^{e}=\left\{u_{1}\left(x_{a}\right), u_{2}\left(x_{a}\right), u_{3}\left(x_{a}\right), u_{4}\left(x_{a}\right), u_{5}\left(x_{a}\right),\right. \\
\left.u_{1}\left(x_{b}\right), u_{2}\left(x_{b}\right), u_{3}\left(x_{b}\right), u_{4}\left(x_{b}\right), u_{5}\left(x_{b}\right)\right\}^{\mathrm{T}}
\end{gathered}
$$

and the force vector $\{\mathbf{F}\}^{e}$ is

$$
\begin{aligned}
& \{\mathbf{F}\}_{\text {thin }}^{e}=\left\{-v_{1}\left(x_{a}\right),-v_{2}\left(x_{a}\right),-v_{3}\left(x_{a}\right),\right. \\
& \left.\quad-v_{4}\left(x_{a}\right), v_{1}\left(x_{b}\right), v_{2}\left(x_{b}\right), v_{3}\left(x_{b}\right), v_{4}\left(x_{b}\right)\right\}^{\mathrm{T}},
\end{aligned}
$$

$$
\begin{aligned}
& \{\mathbf{F}\}_{\text {thick }}^{e}=\left\{-v_{1}\left(x_{a}\right),-v_{2}\left(x_{a}\right),-v_{3}\left(x_{a}\right),-v_{4}\left(x_{a}\right),\right. \\
& \left.\quad-v_{5}\left(x_{a}\right), v_{1}\left(x_{b}\right), v_{2}\left(x_{b}\right), v_{3}\left(x_{b}\right), v_{4}\left(x_{b}\right), v_{5}\left(x_{b}\right)\right\}^{\mathrm{T}} .
\end{aligned}
$$

Mathematically apply the boundary conditions in (11) or (12) to (8) in turn,

$$
\begin{array}{cc}
\{\mathbf{d}\}_{\text {thin }}^{e} & =\left\{e_{j}\right\}, \quad j=1, \ldots, 8, \\
\{\mathbf{d}\}_{\text {thick }}^{e} & =\left\{e_{j}\right\}, \quad j=1, \ldots, 10,
\end{array}
$$

where $\left\{e_{j}\right\}$ is a unit vector with the $j$ th element equal to one, and the corresponding element dynamic stiffness matrix $[\mathbf{k}]^{e}$ is formulated by

$$
\begin{aligned}
& {[\mathbf{k}]_{\text {thin }}^{e}=\left[\begin{array}{llllllll}
\mathbf{F}_{j=1}^{e} & \mathbf{F}_{j=2}^{e} & \mathbf{F}_{j=3}^{e} & \mathbf{F}_{j=4}^{e} & \mathbf{F}_{j=5}^{e} & \mathbf{F}_{j=6}^{e} & \mathbf{F}_{j=7}^{e} & \mathbf{F}_{j=8}^{e}
\end{array}\right]_{\text {thin }},}
\end{aligned}
$$

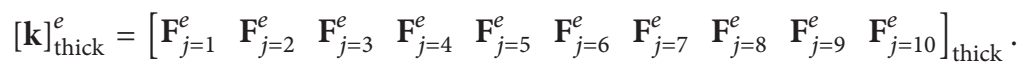

Due to the complexity, (8) is solved computationally using adaptive ordinary differential equations (ODEs) solver COLSYS [33, 34]. The global dynamic stiffness matrix $\mathbf{K}$ of a circular cylindrical shell is assembled in a regular FEM way.

\section{Wittrick-Williams Algorithm}

The dynamic stiffness method is used in conjunction with the Wittrick-Williams (W-W) algorithm. The W-W algorithm merely gives the number of frequencies below $\omega^{*}$ with the expression of

$$
J=J_{0}+J_{K}
$$

where $J$ is the total number of frequencies exceeded by the trial frequency $\omega^{*} ; J_{K}=s\left\{\mathbf{K}\left(\omega^{*}\right)\right\}$ is the sign count of the global dynamic stiffness matrix $\mathbf{K}$ and equal to the number of negative elements on the diagonal of an upper triangular matrix obtained from $\mathbf{K}$ by applying standard Gaussian elimination without row exchange; $J_{0}$ is the number of clamped-end frequencies exceeded by $\omega^{*}$ and can be accumulated from $J_{0}^{e}$ of each shell segment element over the whole meridian as is expressed in

$$
J_{0}=\sum J_{0}^{e}
$$

To solve $J_{0}^{e}$, a substructuring method is employed by taking advantage of the self-adaptability of COLSYS. When computing the dynamic stiffness matrix of a shell segment element, for example, element (e), a submesh will be generated by COLSYS adaptively as is shown in Figure 4.
Applying the Wittrick-Williams algorithm again on the submesh, we have

$$
J_{0}^{e}=\sum J_{0}^{(\widehat{e})}+s\left\{\widehat{\mathbf{K}}\left(\omega^{*}\right)\right\},
$$

where notation “へ” stands for submesh. Since COLSYS is capable of controlling the error tolerance adaptively, the number of clamped-end natural frequencies exceeded by $\omega^{*}$ on the submesh has to be zero, suggesting $J_{0}^{(\widehat{e})}=0$. Otherwise, the clamped-end modes can be enlarged infinitely, resulting in the unsatisfaction of the given error tolerance.

The following will introduce the formulation of $\widehat{\mathbf{K}}\left(\omega^{*}\right)$ in (16). Taking a subelement $(\widehat{e})$ with the coordinates $\left(x_{i}, x_{i+1}\right)$ on element $(e)$, for instance, its corresponding dynamic stiffness matrix $[\widehat{\mathbf{k}}]^{(\widehat{e})}$ can be obtained by linearly combining the eight (or ten) solutions of (8) and (11) or (12). Note that the end displacement and force vectors for this subelement $(\widehat{e})$ are $\{\mathbf{d}\}^{(\widehat{e})}$ and $\{\mathbf{F}\}^{(\widehat{e})}$, respectively, and the subelement stiffness matrix $[\widehat{\mathbf{k}}]^{(\widehat{e})}$ satisfies

$$
[\widehat{\mathbf{k}}]^{(\widehat{e})}\{\mathbf{d}\}^{(\widehat{e})}=\{\mathbf{F}\}^{(\widehat{e})} .
$$

By applying the boundary conditions in (11) or (12) in turn again for thin or moderately thick circular cylindrical shells, (18) is obtained.

$$
[\widehat{\mathbf{k}}]^{(\widehat{e})}[\mathbf{B}]^{(\widehat{e})}=[\mathbf{C}]^{(\widehat{e})},
$$

where

$$
\begin{aligned}
& {[\mathbf{B}]^{(\widehat{e})}}
\end{aligned}
$$

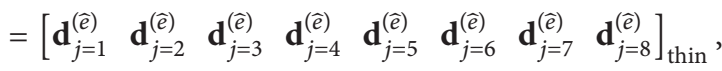

$$
\begin{aligned}
& {[\mathbf{C}]^{(\widehat{e})}} \\
& =\left[\begin{array}{llllllll}
\mathbf{F}_{j=1}^{(\widehat{e})} & \mathbf{F}_{j=2}^{(\widehat{e})} & \mathbf{F}_{j=3}^{(\widehat{e})} & \mathbf{F}_{j=4}^{(\widehat{e})} & \mathbf{F}_{j=5}^{(\widehat{e})} & \mathbf{F}_{j=6}^{(\widehat{e})} & \mathbf{F}_{j=7}^{(\widehat{e})} & \mathbf{F}_{j=8}^{(\widehat{e})}
\end{array}\right]_{\text {thin }}
\end{aligned}
$$




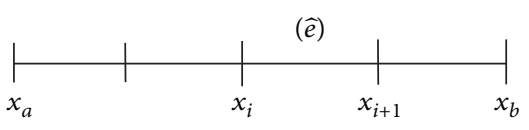

Figure 4: Submesh on element (e).

for thin shells, or

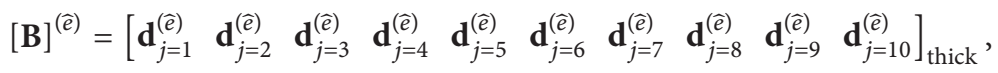

$$
\begin{aligned}
& {[\mathbf{C}]^{(\widehat{e})}=\left[\begin{array}{llllllllll}
\mathbf{F}_{j=1}^{(\widehat{e})} & \mathbf{F}_{j=2}^{(\widehat{e})} & \mathbf{F}_{j=3}^{(\widehat{e})} & \mathbf{F}_{j=4}^{(\widehat{e})} & \mathbf{F}_{j=5}^{(\widehat{e})} & \mathbf{F}_{j=6}^{(\widehat{e})} & \mathbf{F}_{j=7}^{(\widehat{e})} & \mathbf{F}_{j=8}^{(\widehat{e})} & \mathbf{F}_{j=9}^{(\widehat{e})} & \mathbf{F}_{j=10}^{(\widehat{e})}
\end{array}\right]_{\text {thick }}}
\end{aligned}
$$

for moderately thick shells.

Since $J_{0}^{(\widehat{e})}=0,[\mathbf{B}]^{(\widehat{e})}$ is nonsingular (otherwise $\omega^{*}$ is one of clamped-end frequencies, which is conflicted with $J_{0}^{(\widehat{e})}=0$ ), and (21) is obtained.

$$
[\widehat{\mathbf{k}}]^{(\widehat{e})}=[\mathbf{C}]^{(\widehat{e})}\left([\mathbf{B}]^{(\widehat{e})}\right)^{-1}
$$

Thus $\widehat{\mathbf{K}}\left(\omega^{*}\right)$ can be assembled regularly from $[\widehat{\mathbf{k}}]^{(\widehat{e})}$. Based on the above theories, a FORTRAN code was written and used to solve the free vibration of both thin and moderately thick circular cylindrical shells in this paper.

\section{Validation}

In this section, four examples on free vibration of circular cylindrical shells are presented to show the reliability and accuracy of the proposed method in the above sections. Examples in Sections 5.1 and 5.2 are calculated with thin shell theory; in Section 5.3, both thin and moderately thick shell theories are employed to study the free vibration of the same shell, and results are given opposite for comparison; examples in Section 5.4 are performed with the moderately thick shell theory.

5.1. Free Vibration of a Thin Clamped-Clamped Shell. Zhang et al. [28] and Xuebin [29] analysed the natural frequencies of a clamped-clamped (C-C) circular cylindrical shell based on the Flügge thin shell theory by employing the FEM code MSC/NASTRAN and wave propagation approach, respectively. The clamped boundary condition is considered as

$$
u=v=w=\frac{\partial w}{\partial x}=0
$$

The length of the shell is $L=20 \mathrm{~m}$, radius $r=1 \mathrm{~m}$, thickness $h=0.01 \mathrm{~m}$. Density $\rho=7850 \mathrm{~kg} / \mathrm{m}^{3}$, Young's modulus $E=210 \mathrm{GPa}$, Poisson's ratio $v=0.3$. In this example, thin shell theory is used in formulating the dynamic stiffness, and the first nine frequencies are tabulated in Table 1 , where $m$ denotes the number of half-waves along the axial direction.

It can be observed from Table 1 that the frequencies obtained using the present method agree very well with that from the FEM (MSC/NASTRAN) and wave propagation approach. Further investigation into Table 1 shows that the lowest natural frequency of a circular cylindrical usually comes with a relatively larger $n$; that is, $n=2$ in this case.

5.2. Free Vibration of a Thin Clamped-Free Shell. In Sharma [30], the free vibration of a clamped-free (C-F) circular cylindrical shell is investigated based on the Rayleigh-Ritz method and Flügge shell theory. The geometry parameters of the shell are length $L=0.502 \mathrm{~m}$, radius $r=0.0635 \mathrm{~m}$, and thickness $h=0.00163 \mathrm{~m}$; and the material properties are Young's modulus $E=210 \mathrm{GPa}$, Poisson's ratio $\nu=0.28$, and density $\rho=7800 \mathrm{~kg} / \mathrm{m}^{3}$. A thin shell formulation is used in the present dynamic stiffness approach. Table 2 presents the lowest four frequencies of the shell with a relatively larger circumferential wave number $n=5$ and 6 .

It can be concluded that the results agree very well with [30] and the errors are no larger than $0.4 \%$. By comparing the results from the present method and the Rayleigh-Ritz method used in Sharma [30], the reliability of the present method is verified.

5.3. Free Vibration of a Shell with Different Boundary Conditions. Pagani et al. [23] applied the one-dimensional higherorder beam theory and exact dynamic stiffness elements to study the free vibration of solid and thin-walled structures, in which a thin-walled cylinder with a circular cross-section (Figure 5) is analysed. In a recent paper [13], they employed both the radial basis functions (RBFs) method and Carrera Unified Formulation (CUF) to examine the free vibration behaviour of both open and closed thin-walled structures.

The length of the examined cylinder is $L=20 \mathrm{~m}$, outer diameter is $d=2 \mathrm{~m}$, and thickness is $h=0.02 \mathrm{~m}$. The material properties are Young's modulus $E=75 \mathrm{GPa}$, Poisson's ratio $\nu=0.33$, and density $\rho=2700 \mathrm{~kg} / \mathrm{m}^{3}$. Table 3 shows the natural frequencies of this thin-walled cylindrical beam with different boundaries, namely, both ends simply supported (S-S), both ends clamped (C-C), clamped-free $(\mathrm{C}-\mathrm{F})$, and free-free $(\mathrm{F}-\mathrm{F})$. The first and second flexural (bending), shell-like, and torsional natural frequencies are presented. Solutions from the present thin and moderately thick DSM, 2D MSC/NASTRAN, and [13, 23] are given for comparison. 
TABLE 1: The lowest nine frequencies of the C-C circular cylindrical shell $(\mathrm{Hz})$.

\begin{tabular}{lcccc}
\hline Order & $(m, n)$ & Present & Reference [28] & Reference [29] \\
\hline 1 & $(1,2)$ & 12.00 & 12.25 & 12.13 \\
2 & $(1,3)$ & 19.56 & 19.64 & 19.61 \\
3 & $(2,3)$ & 23.10 & 23.18 & 23.28 \\
4 & $(2,2)$ & 27.16 & 27.69 & 28.06 \\
5 & $(1,1)$ & 28.29 & - & 30.09 \\
6 & $(3,3)$ & 31.48 & 31.6 & 31.97 \\
7 & $(1,4)$ & 36.42 & 36.7 & 36.48 \\
8 & $(2,4)$ & 37.28 & 37.55 & 37.38 \\
9 & $(3,4)$ & 39.60 & 39.87 & 39.77 \\
\hline
\end{tabular}

-: not given.

TABLE 2: The lowest four frequencies of the C-F shell with $n=5$ and $6(\mathrm{~Hz})$.

\begin{tabular}{ccccc}
\hline & \multicolumn{2}{c}{$n=5$} & \multicolumn{2}{c}{$n=6$} \\
& Present & Sharma [30] & Present & Sharma [30] \\
\hline 1 & 2361.9 & 2366.6 & 3462.8 & 3469.7 \\
2 & 2402.1 & 2406.4 & 3499.0 & 3505.0 \\
3 & 2505.0 & 2509.1 & 3576.8 & 3580.7 \\
4 & 2706.2 & 2716.0 & 3712.0 & 3716.6 \\
\hline
\end{tabular}

It can be observed that the natural frequencies computed from the present dynamic stiffness method agree very well with that from 2D MSC/NASTRAN code for both thin and moderately thick shell theories. Comparing with the data from the higher-order beam theories $[13,23]$, the present method provides an excellent agreement for flexural and torsional modes. The differences of frequencies for the first and second shell-like modes are larger, and this can be largely attributed that the formulations established in [13, 23] are based on higher-order beam theories. Obviously, better agreement can be achieved if exact shell theories are employed by $[13,23]$. Further investigation by the present DSM approach shows that $n=1$ corresponds to flexural (bending) mode, while $n=0$ and 2 correspond to torsional and shell-like vibration modes, respectively, which demonstrate that the current DSM approach is capable of showing the correct vibration modes as presented in existing literature.

5.4. Free Vibration of a Moderately Thick Shell. Bhimaraddi [32] analysed the free vibration of a simply supported (SS) moderately thick circular cylindrical shell by using the higher-order displacement model with the consideration of in-plane inertia, rotary inertia, and shear effect. In [31], natural frequencies of the circular cylindrical shell subjects to different $h / r$ and $L / r$ are computed and compared with the solutions from 3D elasticity theory. Results from the moderately thick DSM approach are also presented and listed in Table 4. In this example, Poisson's ratio is 0.3 and $\omega_{0}=$ $(\pi / h) \sqrt{G / \rho}$, where $G$ is the shear modulus.

According to Table 4, results from the present method agree very well with those from [31,32], demonstrating the

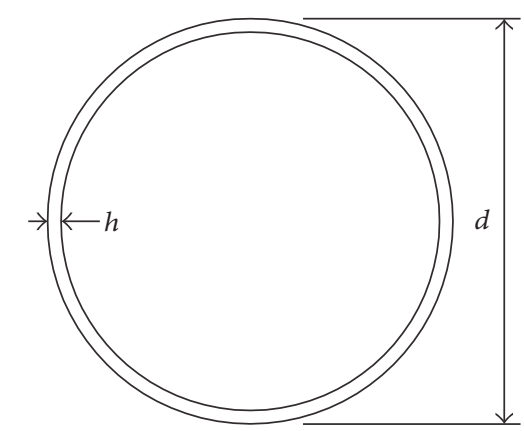

FIGURE 5: The cross-section of a thin-walled cylinder.

high accuracy and adaptability of the present method for circular cylindrical shells with different $h / r$ and $L / r$.

\section{Parametric Study}

6.1. Ratio of Thickness to Radius ( $h / r)$. An important parameter to determine whether a shell is thin or moderately thick is the thickness to radius ratio $(h / r)$. According to the classic shell theory, a critical value for $h / r$ between thin and moderately thick shell theories is 0.05 . In this section, influences of $h / r$ on natural frequencies are investigated extensively from 0.005 to 0.1 for both thin and moderately thick DSM formulations.

6.1.1. Low Order Frequencies. Consider a circular cylindrical shell with $L / r=12$ and Poisson's ratio $v=0.3$. To investigate the differences of natural frequencies between thin and moderately thick DSM formulations, a notation $\eta$ in (23) is introduced as

$$
\eta=\left|\frac{\Omega_{\text {thin }}}{\Omega_{\text {thick }}}-1\right|,
$$

where $\Omega_{\text {thin }}$ and $\Omega_{\text {thick }}$ are the nondimensional frequency parameters defined as

$$
\Omega=\omega r \sqrt{\frac{\rho\left(1-v^{2}\right)}{E}} .
$$

Consequently, $\log _{10}(\eta)$ is the index of difference.

Figure 6 shows the $\log _{10}(\eta)$ for the first frequencies $(m=$ 1) under $n=0,1,2$, and 3 between thin and moderately thick DSM formulations with a variety of boundary conditions, for example, clamped-clamped (C-C), clamped-free (C-F), clamped-simply supported (C-S), and both ends simply supported (S-S). In these cases, $h / r$ is investigated from 0.025 to 0.1 .

If $\log _{10}(\eta) \leq-2$ (which suggests the difference is no larger than 1\%), it can be considered that results from the thin and moderately thick dynamic stiffness formulations are in good agreement with each other. Furthermore, frequencies computed from the moderately thick shell theory can be used as good alternatives to those computed from the thin shell theory. Referring to Figure 6, the following conclusions can be reached: 
TABLE 3: Natural frequencies of the thin-walled cylinder with different boundary conditions $(\mathrm{Hz})$.

\begin{tabular}{|c|c|c|c|c|c|c|c|}
\hline $\mathrm{BCs}$ & Model & I flexural & II flexural & I shell-like & II shell-like & I torsional & II torsional \\
\hline \multirow{5}{*}{ S-S } & Present thin & 14.025 & 51.506 & 14.851 & 22.931 & 80.790 & 161.579 \\
\hline & Present thick & 14.021 & 51.502 & 14.828 & 22.877 & 80.792 & 161.585 \\
\hline & NAS2D [23] & 13.978 & 51.366 & 14.913 & 22.917 & 80.415 & 160.810 \\
\hline & Reference [23] & 14.022 & 51.503 & 18.405 & 25.460 & 80.786 & 161.573 \\
\hline & Reference [13] & 14.294 & 51.567 & 18.608 & 25.574 & 80.639 & 162.551 \\
\hline \multirow{5}{*}{$\mathrm{C}-\mathrm{C}$} & Present thin & 28.577 & 69.110 & 17.350 & 30.239 & 80.790 & 161.579 \\
\hline & Present thick & 28.574 & 69.105 & 17.327 & 30.193 & 80.792 & 161.585 \\
\hline & NAS2D [23] & 28.498 & 68.960 & 17.396 & 30.225 & 80.415 & 160.810 \\
\hline & Reference [23] & 28.576 & 69.110 & 20.484 & 32.222 & 80.786 & 161.573 \\
\hline & Reference [13] & 28.354 & 69.096 & 20.463 & 31.974 & 80.838 & 161.579 \\
\hline \multirow{5}{*}{$\mathrm{C}-\mathrm{F}$} & Present thin & 5.081 & 29.092 & 14.153 & 17.427 & 40.395 & 121.184 \\
\hline & Present thick & 5.076 & 29.086 & 14.140 & 17.370 & 40.396 & 121.188 \\
\hline & NAS2D [23] & 5.059 & 29.001 & 14.235 & 17.435 & 40.209 & 120.620 \\
\hline & Reference [23] & 5.077 & 29.090 & 23.069 & 25.239 & 40.394 & 121.181 \\
\hline & Reference [13] & 5.047 & 29.002 & 23.003 & 24.979 & 40.431 & 121.203 \\
\hline \multirow{5}{*}{ F-F } & Present thin & 30.939 & 77.045 & 14.036 & 14.105 & 80.788 & 161.576 \\
\hline & Present thick & 30.931 & 77.038 & 14.032 & 14.076 & 80.792 & 161.585 \\
\hline & NAS2D [23] & 30.829 & 76.806 & 14.129 & 14.171 & 80.415 & 160.810 \\
\hline & Reference [23] & 30.932 & 77.043 & 22.987 & 23.053 & 80.789 & 161.577 \\
\hline & Reference [13] & 30.945 & 77.052 & 22.864 & 23.048 & 80.787 & 161.592 \\
\hline
\end{tabular}

TABLE 4: Lowest natural frequencies $\left(\times \omega_{0}\right)$ of a moderately thick circular cylindrical shell with different circumferential wave numbers $n$.

\begin{tabular}{lccccccccc}
\hline \multirow{2}{*}{$L / r$} & & \multicolumn{3}{c}{$h / r=0.12$} & & \multicolumn{2}{c}{$h / r=0.18$} \\
& & $n=1$ & $n=2$ & $n=3$ & $n=4$ & $n=1$ & $n=2$ & $n=3$ & $n=4$ \\
\hline \multirow{2}{*}{2} & Present & 0.03724 & 0.02349 & 0.02449 & 0.03664 & 0.05632 & 0.03893 & 0.04939 & 0.07726 \\
& Reference [31] & 0.03730 & 0.02359 & 0.02462 & 0.03686 & 0.05652 & 0.03929 & 0.04996 & 0.07821 \\
& Reference [32] & 0.03730 & 0.02365 & 0.02470 & 0.03687 & 0.05653 & 0.03944 & 0.05009 & 0.07833 \\
\hline \multirow{3}{*}{1} & Present & 0.05861 & 0.04969 & 0.04765 & 0.05508 & 0.09420 & 0.08501 & 0.08995 & 0.11056 \\
& Reference [31] & 0.05853 & 0.04978 & 0.04789 & 0.05545 & 0.09402 & 0.08545 & 0.09093 & 0.11205 \\
& Reference [32] & 0.05856 & 0.04986 & 0.04799 & 0.05550 & 0.09409 & 0.08562 & 0.09109 & 0.11202 \\
\hline \multirow{3}{*}{0.5} & Present & 0.10087 & 0.10238 & 0.10658 & 0.11463 & 0.18915 & 0.19406 & 0.20451 & 0.22180 \\
& Reference [31] & 0.10057 & 0.10234 & 0.10688 & 0.11528 & 0.18894 & 0.19467 & 0.20616 & 0.22450 \\
& Reference [32] & 0.10047 & 0.10224 & 0.10674 & 0.11508 & 0.18832 & 0.19403 & 0.20544 & 0.22361 \\
\hline \multirow{2}{*}{0.25} & Present & 0.27310 & 0.27650 & 0.28219 & 0.29020 & 0.49751 & 0.50299 & 0.51216 & 0.52506 \\
& Reference [31] & 0.27491 & 0.27849 & 0.28447 & 0.29287 & 0.50338 & 0.50937 & 0.51934 & 0.53325 \\
& Reference [32] & 0.27286 & 0.27641 & 0.28233 & 0.29064 & 0.49818 & 0.50418 & 0.51416 & 0.52808 \\
\hline
\end{tabular}

(1) For low order frequencies, good agreement is reached between thin and moderately thick dynamic stiffness formulations with $h / r$ varying from 0.025 to 0.1 . The smaller the $h / r$, the smaller the difference.

(2) The difference between frequencies from thin and moderately thick DSM formulations increases with the increase of $n$, as $\log _{10}(\eta)$ always has a smaller magnitude under $n=0$ than those under $n=1,2$, and 3.

(3) It can be argued that the difference is insensitive to boundary conditions. The differences are roughly within $10^{-5} \sim 10^{-2}$ in this investigation and the trends of curves are similar for different boundary conditions.

6.1.2. High Order Frequencies. Investigation on the differences between high order frequencies computed from the thin and moderately thick DSM formulations is examined in this subsection. Since it is known that the DSM formulation is not sensitive to boundary conditions [26], a C-S circular cylindrical shell is studied. The geometry and material properties are the same to the example in Section 6.1.1 if not specifically stated.

For high order frequencies, $h / r$ is investigated from very small value 0.005 to a relatively large value 0.1 , and the first 


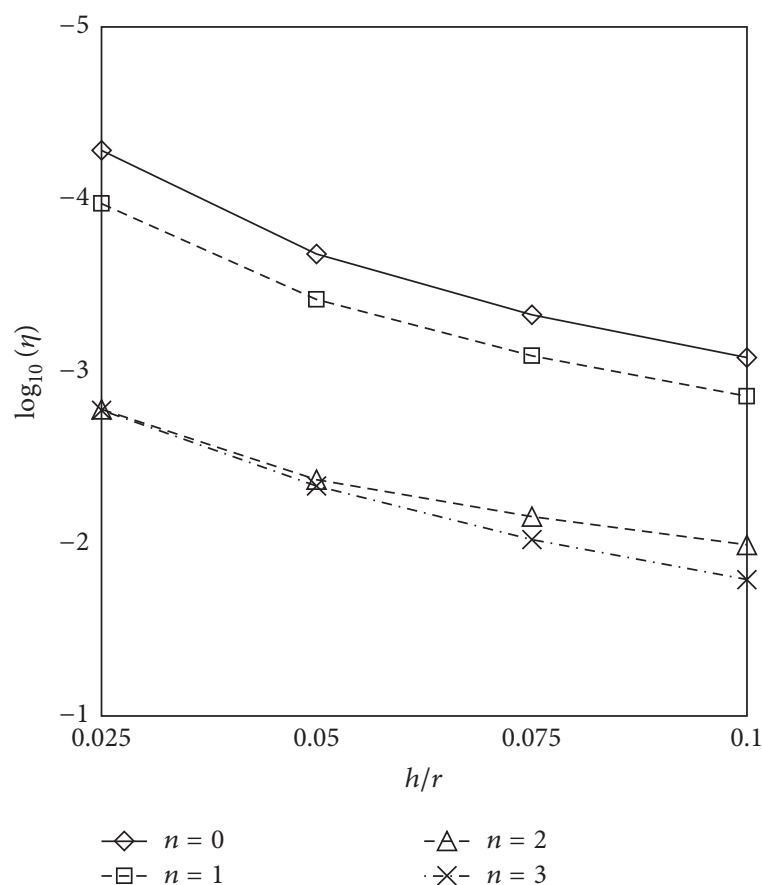

(a) $\mathrm{C}-\mathrm{C}$

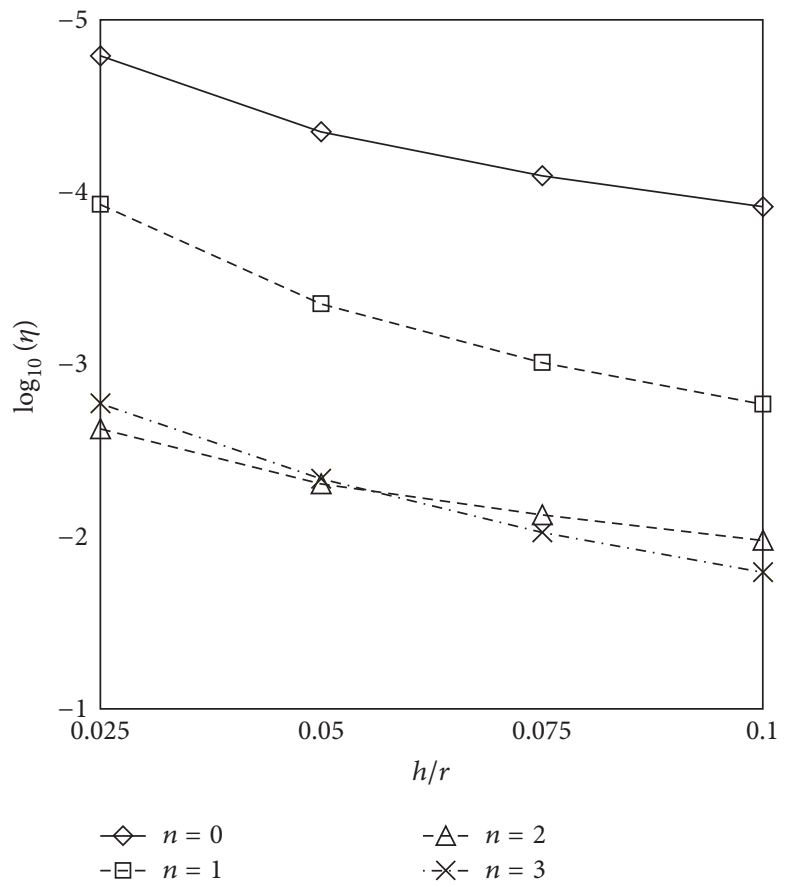

(c) C-S

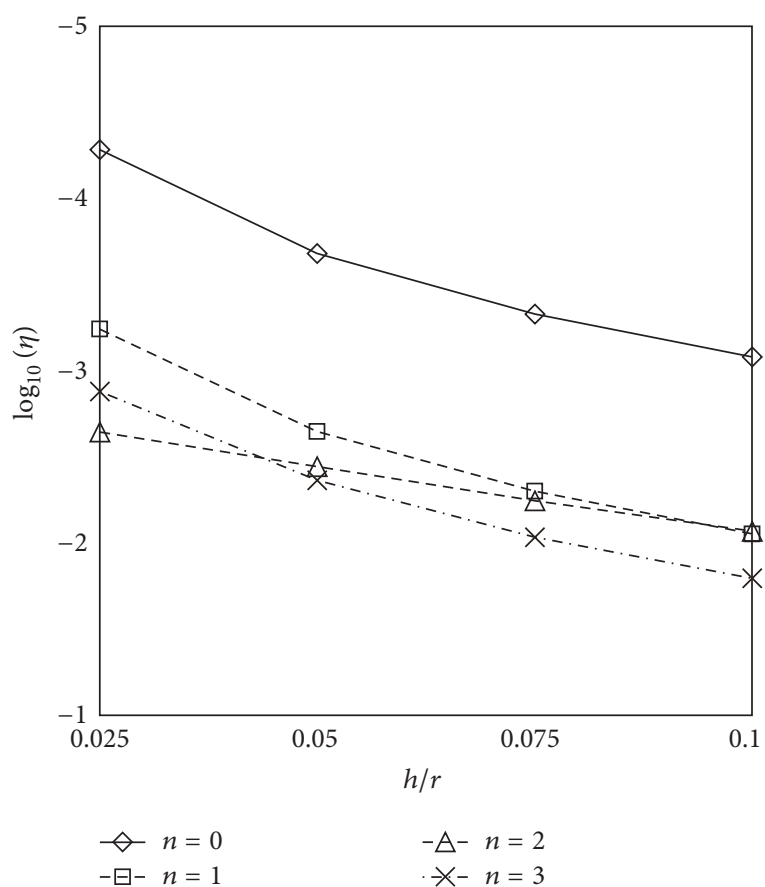

(b) C-F

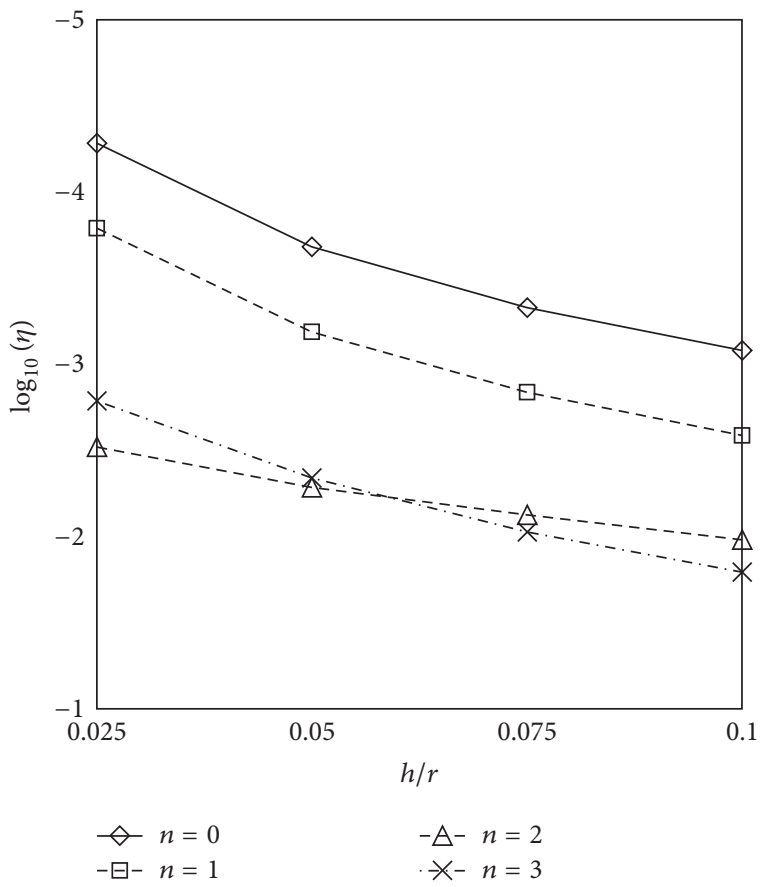

(d) S-S

FIgURE 6: The differences of low order frequencies between thin and moderately thick DSM formulations.

ten frequencies under $n$ ranging from 0 to 10 from both thin and moderately thick DSM formulations are computed and compared. The differences are shown in Figure 7 and concluded as follows:

(1) When $h / r \leq 0.025, \log _{10}(\eta)$ is smaller than $10^{-2}$ most of the time, which suggests that frequencies computed from moderately thick shell theory are well compatible with those from thin shell theory. No shear locking is observed.

(2) When $h / r \geq 0.05$, only frequencies with a small $n$ (e.g., $n \leq 2)$ produce an acceptable difference $\left(\sim 10^{-2}\right)$. With the increase of $n$, the difference becomes quite noticeable. The higher the $n$, the more obvious the difference. 


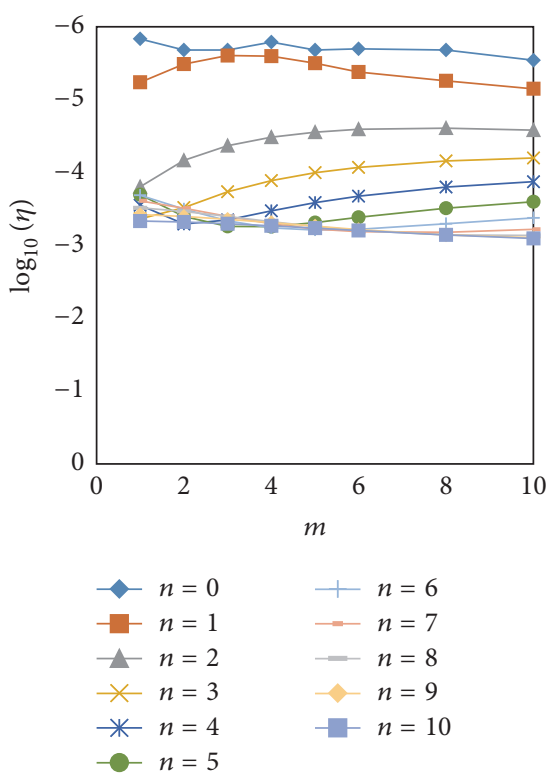

(a) $h / r=0.005$

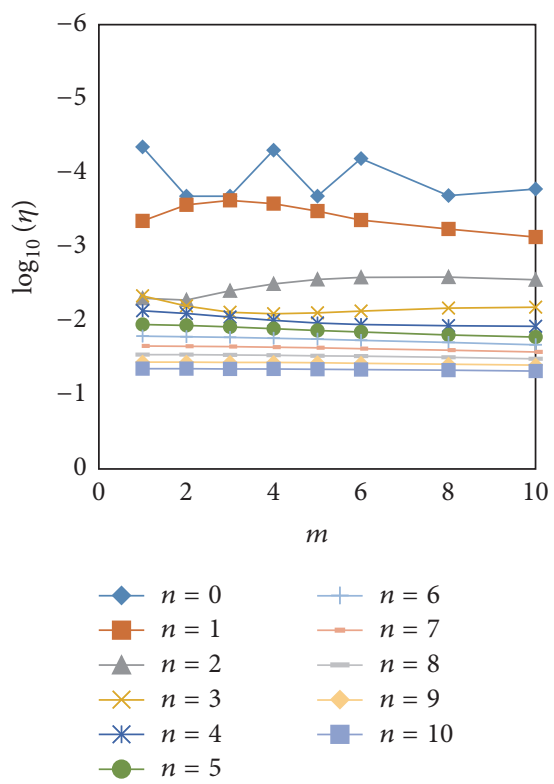

(d) $h / r=0.05$

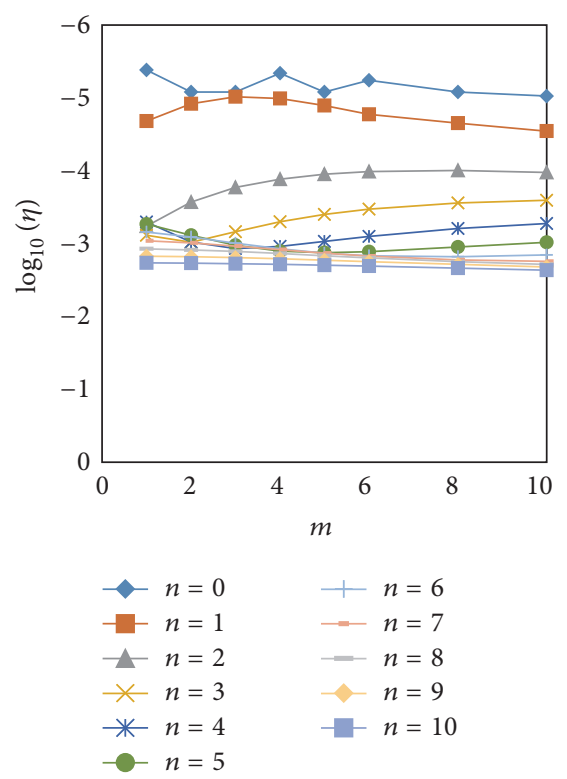

(b) $h / r=0.01$
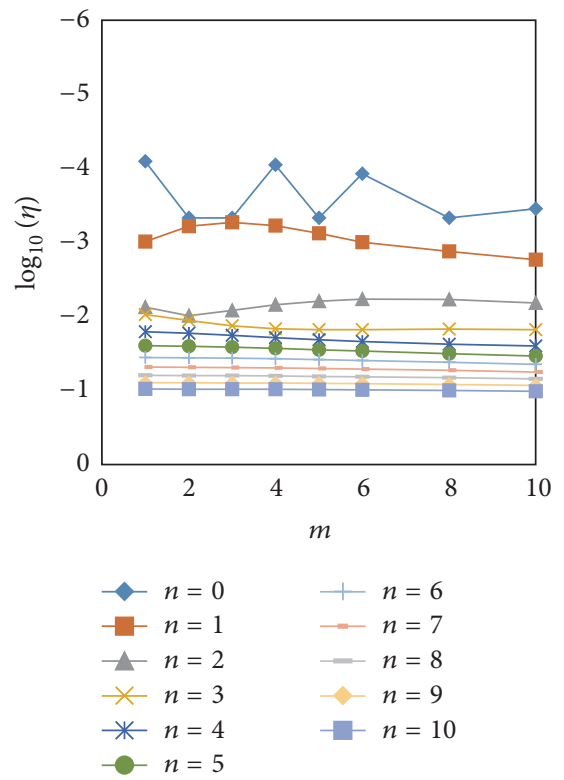

(e) $h / r=0.075$
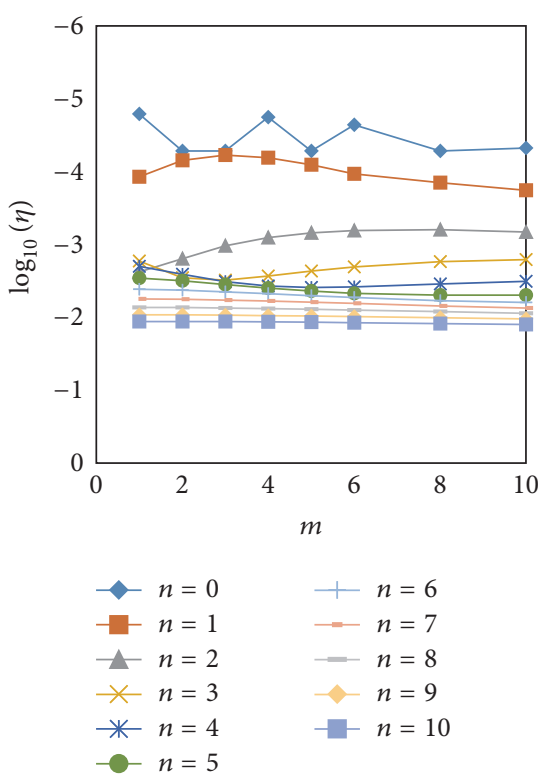

(c) $h / r=0.025$
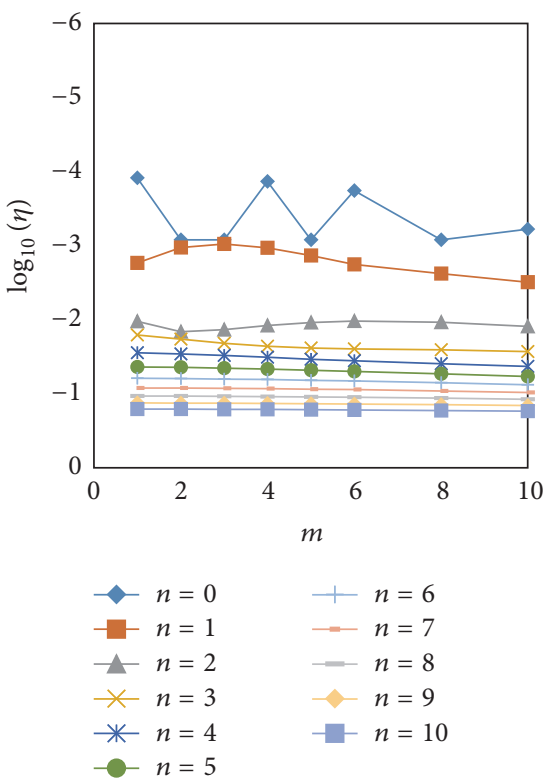

(f) $h / r=0.1$

FIGURE 7: The differences for frequencies between thin and moderately thick C-S circular cylindrical shell theories.

(3) The order $m$ under a specific circumferential wave number $n$ does not have a significant impact on the differences, as most curves in Figure 7 are largely flat and stable with the increase of $m$.

6.2. Ratio of Length to Radius $(L / r)$. Parameter $L / r$ controls the relative length of a circular cylindrical shell. A larger $L / r$ indicates a long shell, and vice versa. In this subsection, the frequency trends and the compatibility between thin and moderately thick DSM formulations with respect to $L / r$ are studied. In this subsection, a C-C circular cylindrical shell with $v=0.3$ is examined. Figure 8 plots the trends of the first frequency parameter $\Omega$ under each $n$ with $L / r$ from 0.2 to 10 .

According to Figure 8, for a typical long circular cylindrical shell (e.g., $L / r=10$ ), the lowest natural frequency is obtained with $n=2$; with the decrease of $L / r$, the circumferential wave number $n$ corresponding to the lowest natural frequency slightly increases to $n=3$ or 4 ; when $L / r$ is very small (e.g., $L / r=0.2$ and the shell is actually degraded to a ring), the first natural frequency under each $n$ increases monotonically and the smallest is reached at $n=0$. The trends shown by Figure 8 are also in good agreement with other researchers' work, 


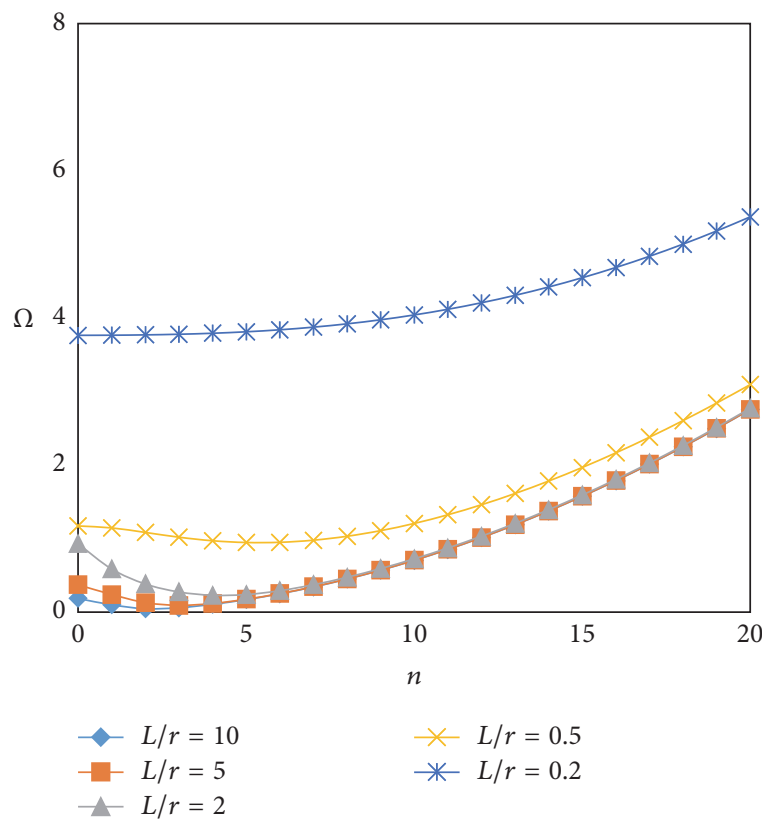

(a) $h / r=0.025$, moderately thick shell

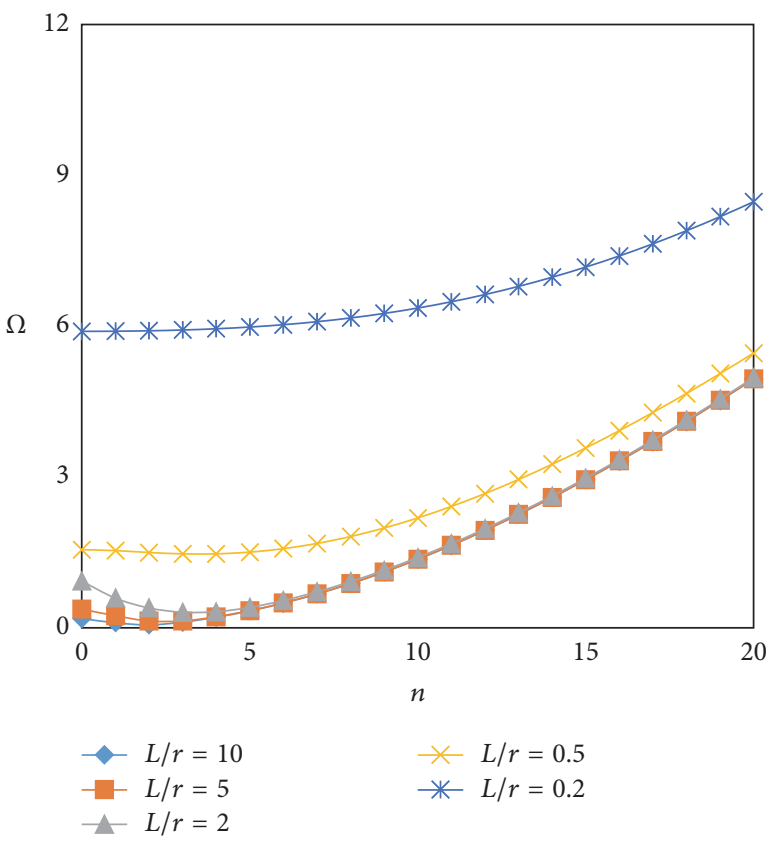

(c) $h / r=0.05$, moderately thick shell

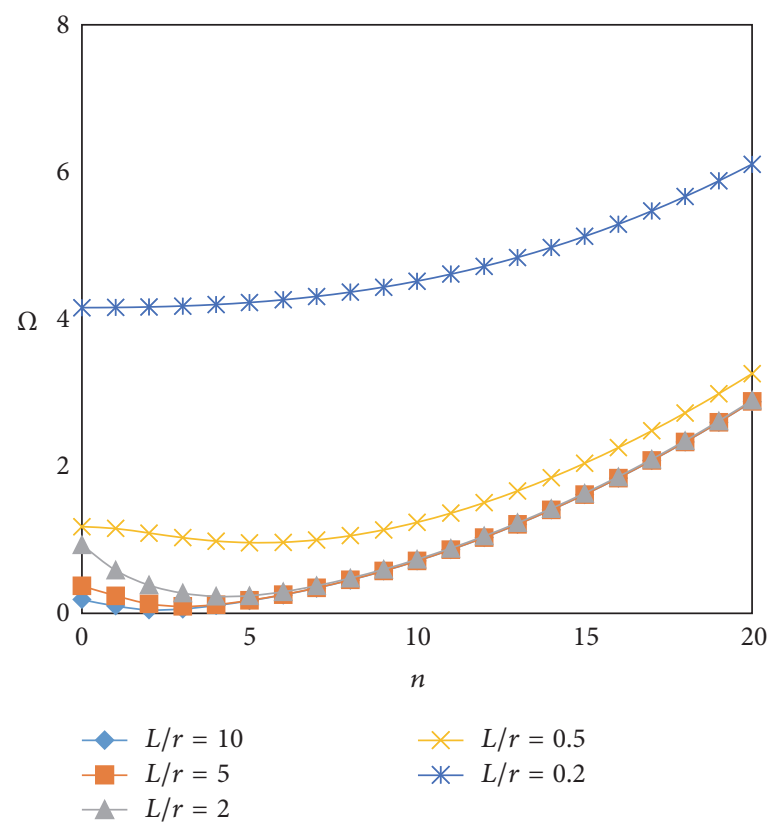

(b) $h / r=0.025$, thin shell

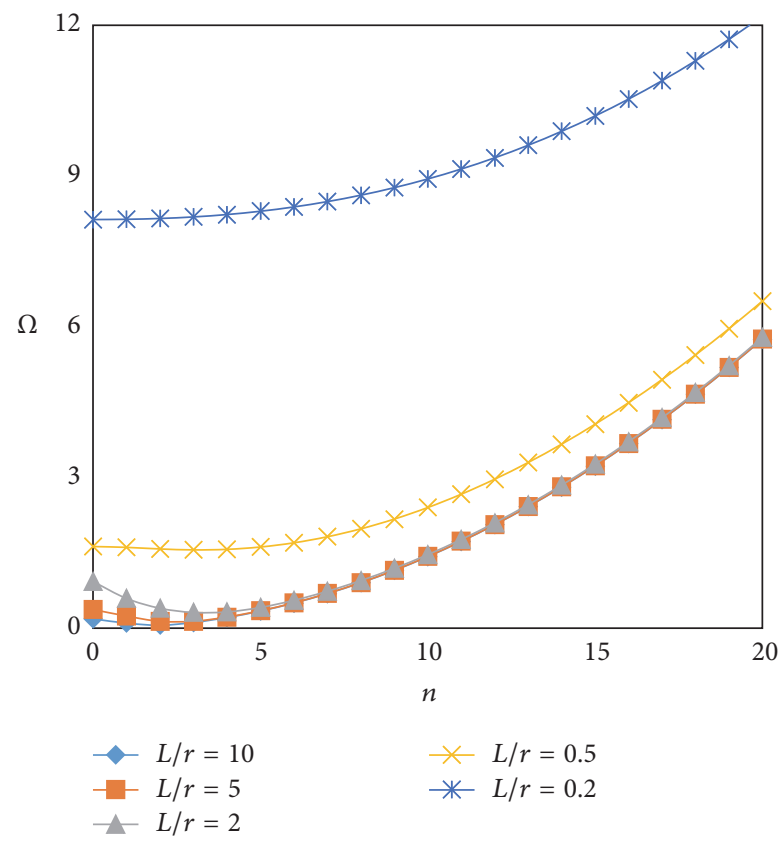

(d) $h / r=0.05$, thin shell

FIGURE 8: The trends of the first natural frequency parameters $\Omega$ under different $n$.

for example, Markuš [35]. These obtained trends are applicable to both thin and moderately thick DSM formulations.

Figure 9 shows the differences of the first frequencies under each $n$ computed from the thin and moderately thick DSM formulations of the circular cylindrical shell with $h / r=$ 0.025 and 0.05 . It can be understood from Figure 9 that (1) the smaller the $h / r$, the smaller the difference; (2) the larger the $L / r$, the smaller the difference; (3) conditions of $h / r \leq 0.025$ and $n \leq 10$ need to be satisfied and a minor difference $\left(\log _{10}(\eta) \leq 10^{-2}\right)$ is required for a normal length shell; that is, $L / r \geq 2$.

\section{Conclusions}

This paper conducts comparison study on the frequencies from thin and moderately thick circular cylindrical shells computed using the DSM formulations, and comprehensive parametric studies on $h / r$ and $L / r$ are performed. Based on these investigations, conclusions are outlined as follows:

(1) Natural frequencies computed from the moderately thick DSM formulations can be considered as satisfactory alternatives to those from thin DSM formulations when $h / r \leq 0.025$ and $L / r \geq 2$ 


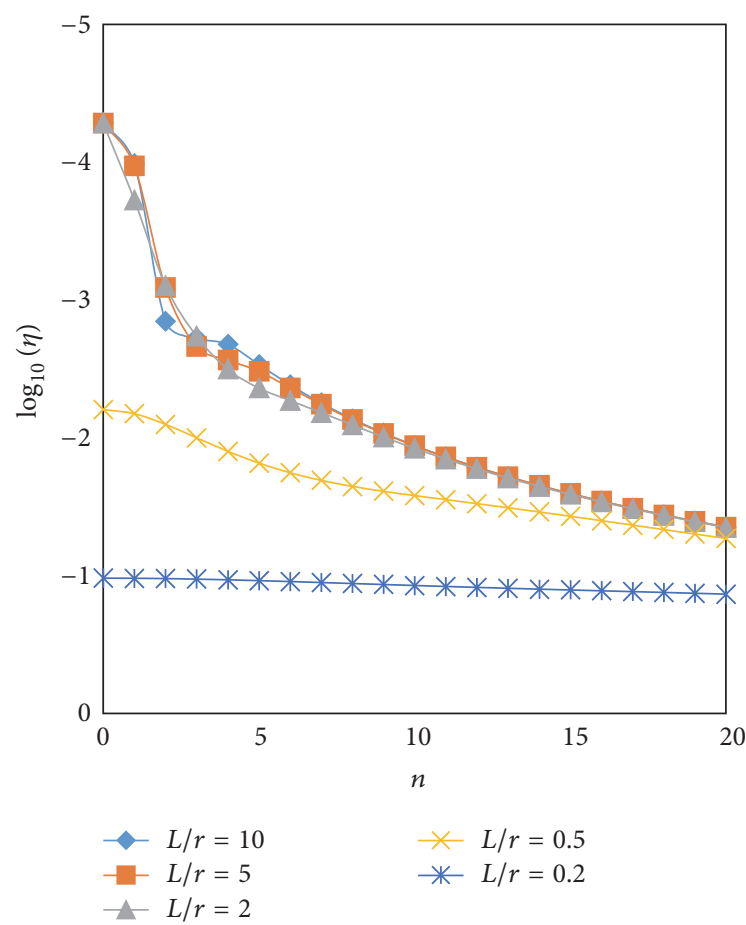

(a) $h / r=0.025$

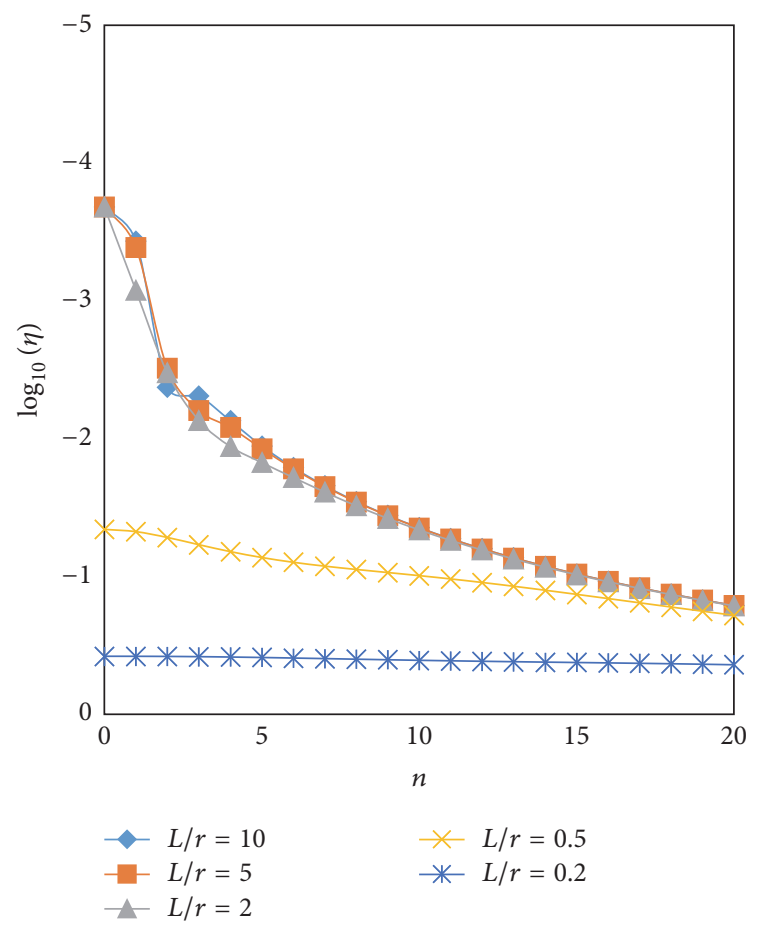

(b) $h / r=0.05$

FIGURE 9: Differences between the first frequencies under each $n$ with different $L / r$.

(differences are around or smaller than $10^{-2}$ ). Since the DSM is exact, no shear locking is observed.

(2) Differences between frequencies from thin and moderately thick DSM formulations change with a variety of factors. The smaller $h / r$, the smaller the difference; the smaller $L / r$, the larger the difference; the larger the $n$, the larger the difference.

It is proved that moderately thick DSM formulations can be used to analyse the free vibration of normal length thin circular cylindrical shells without shear locking, demonstrating that the applicable range of moderately thick DSM formulations is expanded. These merits should be attributed to that the dynamic stiffness method is an exact method.

Though this comparison study is performed on circular cylindrical shells, similar conclusions can be extended to the free vibration of other shaped shells of revolution.

\section{Appendix}

$$
\begin{aligned}
& \left\{\mathbf{u}_{\text {thin }}=\left\{\begin{array}{l}
u_{1}=u_{n} \\
u_{2}=v_{n} \\
u_{3}=w_{n} \\
u_{4}=w_{n}^{\prime},
\end{array}\right.\right. \\
& \left\{\begin{array}{l}
v_{\text {thin }}= \\
v_{1}=\frac{r \pi E h}{1-v^{2}}\left[\nu \frac{n}{r} v_{n}+v \frac{1}{r} w_{n}+u_{n}^{\prime}\right] \\
v_{2}=\frac{r \pi E h}{2(1+\nu)}\left[-\frac{n}{r} u_{n}+\left(1+\frac{h^{2}}{12 r^{2}}\right) v_{n}^{\prime}+\frac{n h^{2}}{6 r^{2}} w_{n}^{\prime}\right] \\
v_{3}=\frac{r \pi E h}{2\left(1-v^{2}\right)}\left[\frac{n h^{2}}{6 r^{2}} v_{n}^{\prime}+(2-v) \frac{n^{2} h^{2}}{6 r^{2}} w_{n}^{\prime}-\frac{h^{2}}{6} w_{n}^{\prime \prime \prime}\right] \\
v_{4}=\frac{r \pi E h}{12\left(1-v^{2}\right)}\left[-\nu \frac{n h^{2}}{r^{2}} v_{n}-v \frac{n^{2} h^{2}}{r^{2}} w_{n}+h^{2} w_{n}^{\prime \prime}\right],
\end{array}\right.
\end{aligned}
$$




$$
\begin{aligned}
& \{\mathbf{u}\}_{\text {thick }}=\left\{\begin{array}{l}
u_{1}=u_{n} \\
u_{2}=v_{n} \\
u_{3}=w_{n} \\
u_{4}=\psi_{x n} \\
u_{5}=\psi_{\theta n},
\end{array}\right. \\
& \{\mathbf{v}\}_{\text {thick }}=\left\{\begin{array}{l}
v_{1}=\frac{r \pi E h}{1-v^{2}}\left[u_{n}^{\prime}+\nu \frac{n}{r} v_{n}+\frac{v}{r} w_{n}\right] \\
v_{2}=\frac{1}{2} \frac{r \pi E h}{1+v}\left[-\frac{n}{r} u_{n}+\left(1+\frac{h^{2}}{12 r^{2}}\right) v_{n}^{\prime}-\frac{n h^{2}}{12 r^{2}} \psi_{x n}+\frac{h^{2}}{12 r^{2}} \psi_{\theta n}^{\prime}\right] \\
v_{3}=r \pi \bar{\kappa} G h\left[w_{n}^{\prime}+\psi_{x n}\right] \\
v_{4}=\frac{h^{2}}{12} \frac{r \pi E h}{1-v^{2}}\left[\psi_{x n}^{\prime}+v_{\frac{n}{r}} \psi_{\theta n}\right] \\
v_{5}=\frac{h^{2}}{24} \frac{r \pi E h}{1+v}\left[\frac{v_{n}^{\prime}}{r}-\frac{n}{r} \psi_{x n}+\psi_{\theta n}^{\prime}\right],
\end{array}\right.
\end{aligned}
$$

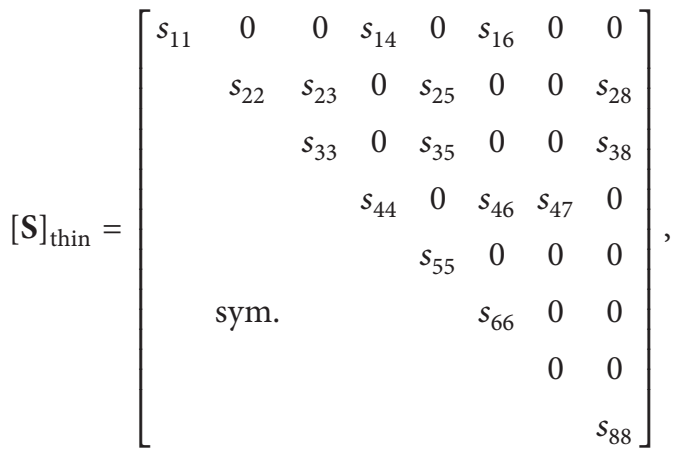

where nonzero elements in the upper triangular domain are

$$
s_{35}=-\frac{\nu}{r}
$$

$$
\begin{aligned}
& s_{11}=\rho \pi r h \omega^{2}-\frac{\pi E h n^{2}}{2 r(1-v)}+\frac{6 n^{2}}{\left(12 r^{2}+h^{2}\right)} \\
& s_{14}=-\frac{n^{2} h^{3} \pi E}{\left(12 r^{2}+h^{2}\right)(1+\nu)}, \\
& s_{16}=\frac{12 n r}{12 r^{2}+h^{2}}, \\
& s_{22}=\rho \pi r h \omega^{2}-\frac{n^{2} \pi E h\left(12 r^{2}+h^{2}\right)}{12 r^{3}}, \\
& s_{23}=-\pi E h \frac{12 n r^{2}+n^{3} h^{2}}{12 r^{3}}, \\
& s_{25}=-\nu \frac{n}{r}, \\
& s_{28}=\nu \frac{n}{r^{2}}, \\
& s_{33}=\rho h r \pi \omega^{2}-\pi E \frac{h}{r}-\frac{n^{4} h^{3} \pi E}{12 r^{3}},
\end{aligned}
$$$$
s_{38}=\frac{v n^{2}}{r^{2}},
$$$$
s_{44}=-\frac{2 n^{2} h^{3} r \pi E}{\left(12 r^{2}+h^{2}\right)(1+v)},
$$$$
s_{46}=-\frac{2 n h^{2}}{12 r^{2}+h^{2}},
$$$$
s_{47}=1 \text {, }
$$$$
s_{55}=\frac{1-v^{2}}{r \pi E h},
$$$$
s_{66}=\frac{24 r(1+v)}{\pi E h\left(12 r^{2}+h^{2}\right)},
$$$$
s_{88}=\frac{12\left(1-v^{2}\right)}{r \pi E h^{3}},
$$

$[\mathbf{S}]_{\text {thick }}$ 


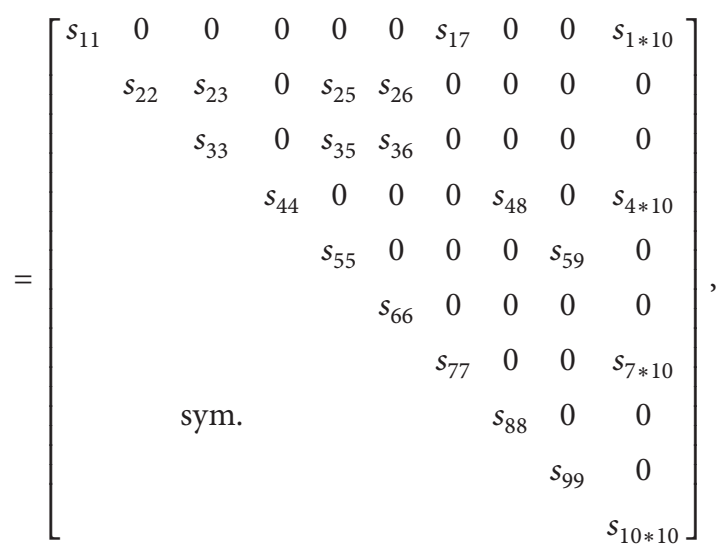

where nonzero elements in the upper triangular domain are

$$
\begin{aligned}
& s_{11}=r \pi \rho h \omega^{2}, \\
& s_{17}=\frac{n}{r}, \\
& s_{1 * 10}=-\frac{n}{r^{2}}, \\
& s_{22}=\pi\left(r \rho h \omega^{2}-\frac{\bar{\kappa} G h}{r}-\frac{n^{2} E h}{r}\right), \\
& s_{23}=-\frac{n \pi}{r}(E h+\bar{\kappa} G h) \text {, } \\
& s_{25}=\pi \bar{\kappa} G h, \\
& s_{26}=-v \frac{n}{r}, \\
& s_{33}=\pi\left(r \rho h \omega^{2}-\frac{E h}{r}-\frac{n^{2} \bar{\kappa} G h}{r}\right), \\
& s_{35}=n \pi \bar{\kappa} G h \text {, } \\
& s_{36}=-\frac{\nu}{r}, \\
& s_{44}=r \pi \rho \omega^{2} \frac{h^{3}}{12}, \\
& s_{48}=-1 \text {, } \\
& s_{4 * 10}=\frac{n}{r}, \\
& s_{55}=\pi\left(r \rho \omega^{2} \frac{h^{3}}{12}-\frac{n^{2} E h^{3}}{12 r}-r \bar{\kappa} G h\right) \text {, } \\
& s_{59}=-v \frac{n}{r}, \\
& s_{66}=\frac{1-v^{2}}{r \pi E h}, \\
& s_{77}=\frac{2(1+\nu)}{r \pi E h},
\end{aligned}
$$

$$
\begin{aligned}
s_{7 * 10} & =-\frac{2(1+\nu)}{r^{2} \pi E h}, \\
s_{88} & =\frac{1}{r \pi \bar{\kappa} G h}, \\
s_{99} & =\frac{12\left(1-v^{2}\right)}{r \pi E h^{3}}, \\
s_{10 * 10} & =\frac{2(1+\nu)}{r \pi E h}\left(\frac{12}{h^{2}}+\frac{1}{r^{2}}\right), \\
\bar{\kappa} & =\frac{\pi^{2}}{12} .
\end{aligned}
$$

\section{Competing Interests}

The authors declare that there is no conflict of interests regarding the publication of this paper.

\section{Acknowledgments}

The authors gratefully appreciate the financial support from National Natural Science Foundation of China (51078198), Tsinghua University (2011THZ03), Jiangsu University of Science and Technology (1732921402), and Shuang-Chuang Program of Jiangsu Province for this research.

\section{References}

[1] A. E. H. Love, A Treatise on the Mathematical Theory of Elasticity, Dover, England, UK, 1927.

[2] R. N. Arnold and G. B. Warburton, "The flexural vibrations of thin cylinders," Proceedings of the Institution of Mechanical Engineers, vol. 167, no. 1, pp. 62-80, 1953.

[3] A. W. Leissa, "Vibration of shells," Report 288, National Aeronautics and Space Administration (NASA), Washington, DC, USA, 1973.

[4] N. S. Bardell, J. M. Dunsdon, and R. S. Langley, "On the free vibration of completely free, open, cylindrically curved isotropic shell panels," Journal of Sound and Vibration, vol. 207, no. 5, pp. 647-669, 1997.

[5] D.-Y. Tan, "Free vibration analysis of shells of revolution," Journal of Sound and Vibration, vol. 213, no. 1, pp. 15-33, 1998.

[6] P. M. Naghdi and R. M. Cooper, "Propagation of elastic waves in cylindrical shells, including the effects of transverse shear and rotatory inertia," The Journal of the Acoustical Society of America, vol. 28, no. 1, pp. 56-63, 1956.

[7] I. Mirsky and G. Herrmann, "Nonaxially symmetric motions of cylindrical shells," The Journal of the Acoustical Society of America, vol. 29, pp. 1116-1123, 1957.

[8] F. Tornabene and E. Viola, "2-D solution for free vibrations of parabolic shells using generalized differential quadrature method," European Journal of Mechanics, A/Solids, vol. 27, no. 6, pp. 1001-1025, 2008.

[9] J. L. Mantari, A. S. Oktem, and C. Guedes Soares, "Bending and free vibration analysis of isotropic and multilayered plates and shells by using a new accurate higher-order shear deformation theory," Composites Part B: Engineering, vol. 43, no. 8, pp. 33483360, 2012. 
[10] J. G. Kim, J. K. Lee, and H. J. Yoon, "Free vibration analysis for shells of revolution based on p-version mixed finite element formulation," Finite Elements in Analysis and Design, vol. 95, pp. 12-19, 2015.

[11] F. Tornabene, "Free vibrations of laminated composite doublycurved shells and panels of revolution via the GDQ method," Computer Methods in Applied Mechanics and Engineering, vol. 200, no. 9-12, pp. 931-952, 2011.

[12] F. Tornabene, N. Fantuzzi, and M. Bacciocchi, "The local GDQ method applied to general higher-order theories of doublycurved laminated composite shells and panels: the free vibration analysis," Composite Structures, vol. 116, pp. 637-660, 2014.

[13] A. Pagani, E. Carrera, and A. Ferreira, "Higher-order theories and radial basis functions applied to free vibration analysis of thin-walled beams," Mechanics of Advanced Materials and Structures, vol. 23, no. 9, pp. 1080-1091, 2016.

[14] T. Ye, G. Jin, Y. Chen, and S. Shi, "A unified formulation for vibration analysis of open shells with arbitrary boundary conditions," International Journal of Mechanical Sciences, vol. 81, pp. 42-59, 2014.

[15] T. Ye, G. Jin, S. Shi, and X. Ma, "Three-dimensional free vibration analysis of thick cylindrical shells with general end conditions and resting on elastic foundations," International Journal of Mechanical Sciences, vol. 84, pp. 120-137, 2014.

[16] F. W. Williams and W. H. Wittrick, "An automatic computational procedure for calculating natural frequencies of skeletal structures," International Journal of Mechanical Sciences, vol. 12, no. 9, pp. 781-791, 1970.

[17] W. H. Wittrick and F. W. Williams, "A general algorithm for computing natural frequencies of elastic structures," The Quarterly Journal of Mechanics and Applied Mathematics, vol. 24, pp. 263-284, 1971.

[18] S. Yuan, K. Ye, F. W. Williams, and D. Kennedy, "Recursive second order convergence method for natural frequencies and modes when using dynamic stiffness matrices," International Journal for Numerical Methods in Engineering, vol. 56, no. 12, pp. 1795-1814, 2003.

[19] S. Yuan, K. Ye, and F. W. Williams, "Second order mode-finding method in dynamic stiffness matrix methods," Journal of Sound and Vibration, vol. 269, no. 3-5, pp. 689-708, 2004.

[20] S. Yuan, K. Ye, C. Xiao, F. W. Williams, and D. Kennedy, "Exact dynamic stiffness method for non-uniform Timoshenko beam vibrations and Bernoulli-Euler column buckling," Journal of Sound and Vibration, vol. 303, no. 3-5, pp. 526-537, 2007.

[21] J. R. Banerjee, C. W. Cheung, R. Morishima, M. Perera, and J. Njuguna, "Free vibration of a three-layered sandwich beam using the dynamic stiffness method and experiment," International Journal of Solids and Structures, vol. 44, no. 22-23, pp. 7543-7563, 2007.

[22] H. Su, J. R. Banerjee, and C. W. Cheung, "Dynamic stiffness formulation and free vibration analysis of functionally graded beams," Composite Structures, vol. 106, pp. 854-862, 2013.

[23] A. Pagani, M. Boscolo, J. R. Banerjee, and E. Carrera, "Exact dynamic stiffness elements based on one-dimensional higherorder theories for free vibration analysis of solid and thinwalled structures," Journal of Sound and Vibration, vol. 332, no. 23, pp. 6104-6127, 2013.

[24] N. El-Kaabazi and D. Kennedy, "Calculation of natural frequencies and vibration modes of variable thickness cylindrical shells using the Wittrick-Williams algorithm," Computers and Structures, vol. 104-105, pp. 4-12, 2012.
[25] X. Chen, Research on dynamic stiffness method for free vibration of shells of revolution [M.S. thesis], Tsinghua University, 2009 (Chinese).

[26] X. Chen and K. Ye, "Free Vibration Analysis for Shells of Revolution Using an Exact Dynamic Stiffness Method," Mathematical Problems in Engineering, vol. 2016, Article ID 4513520, 12 pages, 2016.

[27] X. Chen and K. Ye, "Analysis of free vibration of moderately thick circular cylindrical shells using the dynamic stiffness method," Engineering Mechanics, vol. 33, no. 9, pp. 40-48, 2016 (Chinese).

[28] X. M. Zhang, G. R. Liu, and K. Y. Lam, "Vibration analysis of thin cylindrical shells using wave propagation approach," Journal of Sound and Vibration, vol. 239, no. 3, pp. 397-403, 2001.

[29] L. Xuebin, "Study on free vibration analysis of circular cylindrical shells using wave propagation," Journal of Sound and Vibration, vol. 311, no. 3-5, pp. 667-682, 2008.

[30] C. B. Sharma, "Calculation of natural frequencies of fixed-free circular cylindrical shells," Journal of Sound and Vibration, vol. 35, no. 1, pp. 55-76, 1974.

[31] A. E. Armenakas, D. C. Gazis, and G. Herrmann, Free Vibrations of Circular Cylindrical Shells, Pergamon Press, Oxford, UK, 1969.

[32] A. Bhimaraddi, "A higher order theory for free vibration analysis of circular cylindrical shells," International Journal of Solids and Structures, vol. 20, no. 7, pp. 623-630, 1984.

[33] U. Ascher, J. Christiansen, and R. D. Russell, "Algorithm 569, COLSYS: collocation software for boundary value ODEs [D2]," ACM Transactions on Mathematical Software, vol. 7, no. 2, pp. 223-229, 1981.

[34] U. Ascher, J. Christiansen, and R. D. Russell, "Collocation software for boundary-value ODEs," ACM Transactions on Mathematical Software, vol. 7, no. 2, pp. 209-222, 1981.

[35] Š. Markuš, The Mechanics of Vibrations of Cylindrical Shells, Elsevier Science Limited, 1988. 


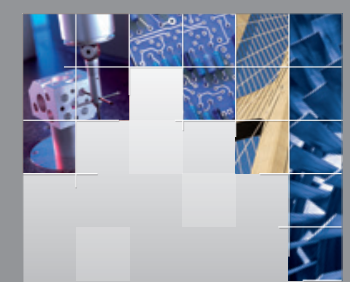

\section{Enfincering}
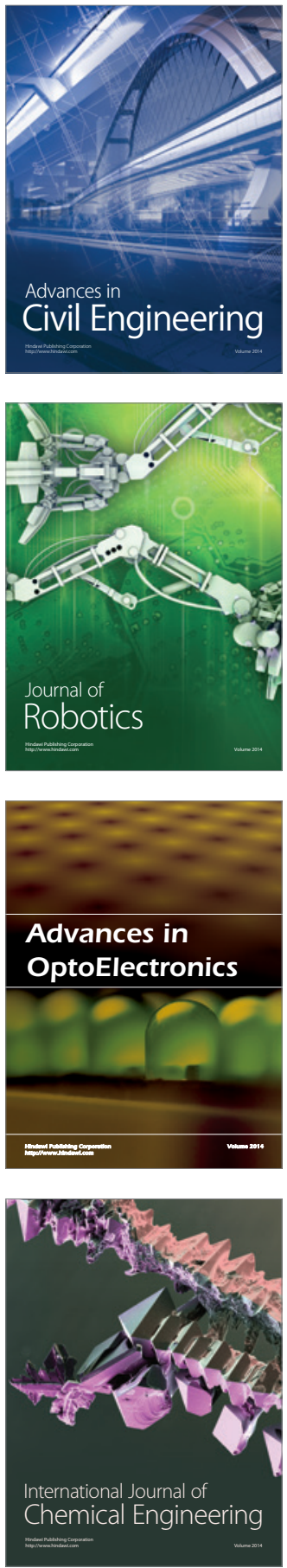

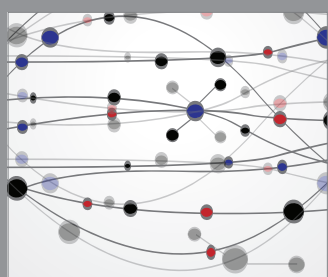

The Scientific World Journal

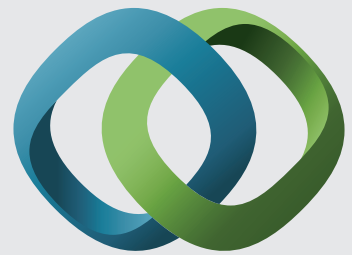

\section{Hindawi}

Submit your manuscripts at

http://www.hindawi.com
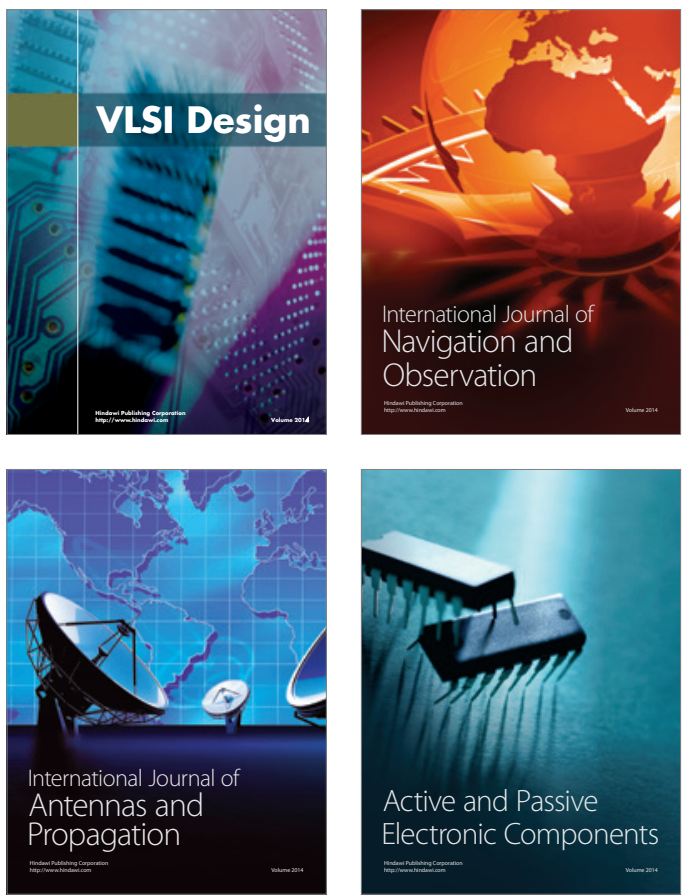
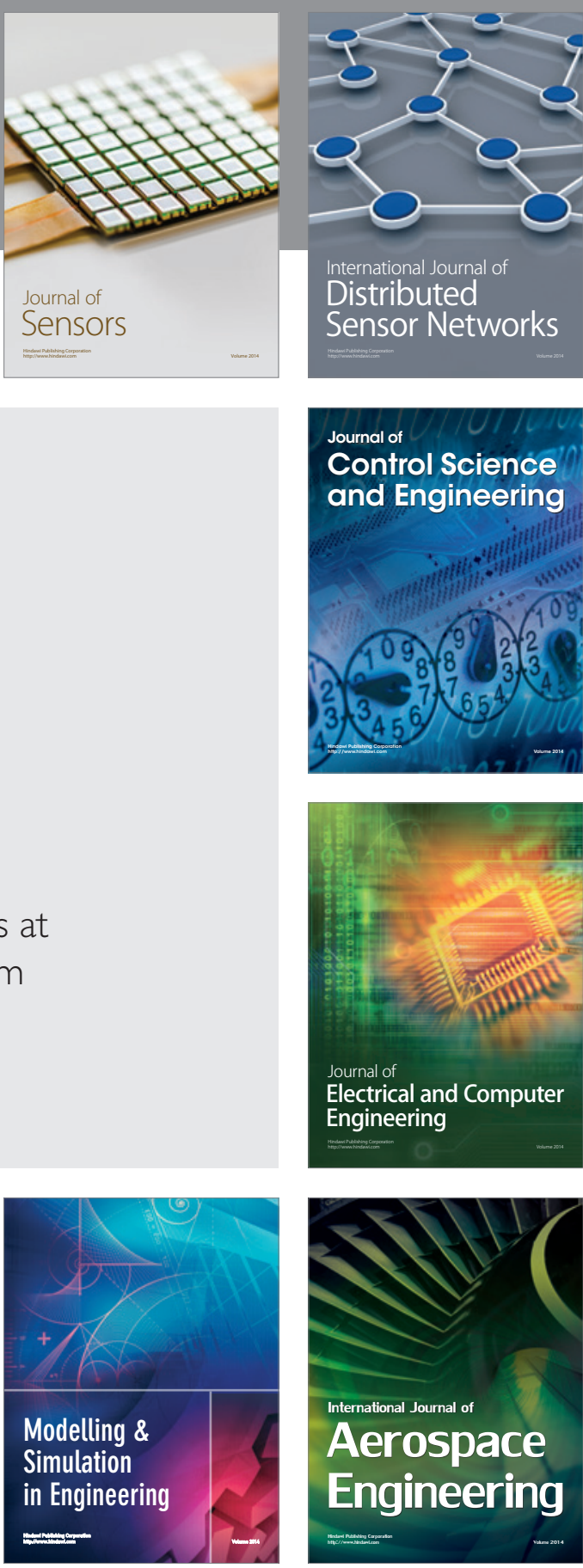

International Journal of

Distributed

Sensor Networks

Journal of

Control Science

and Engineering
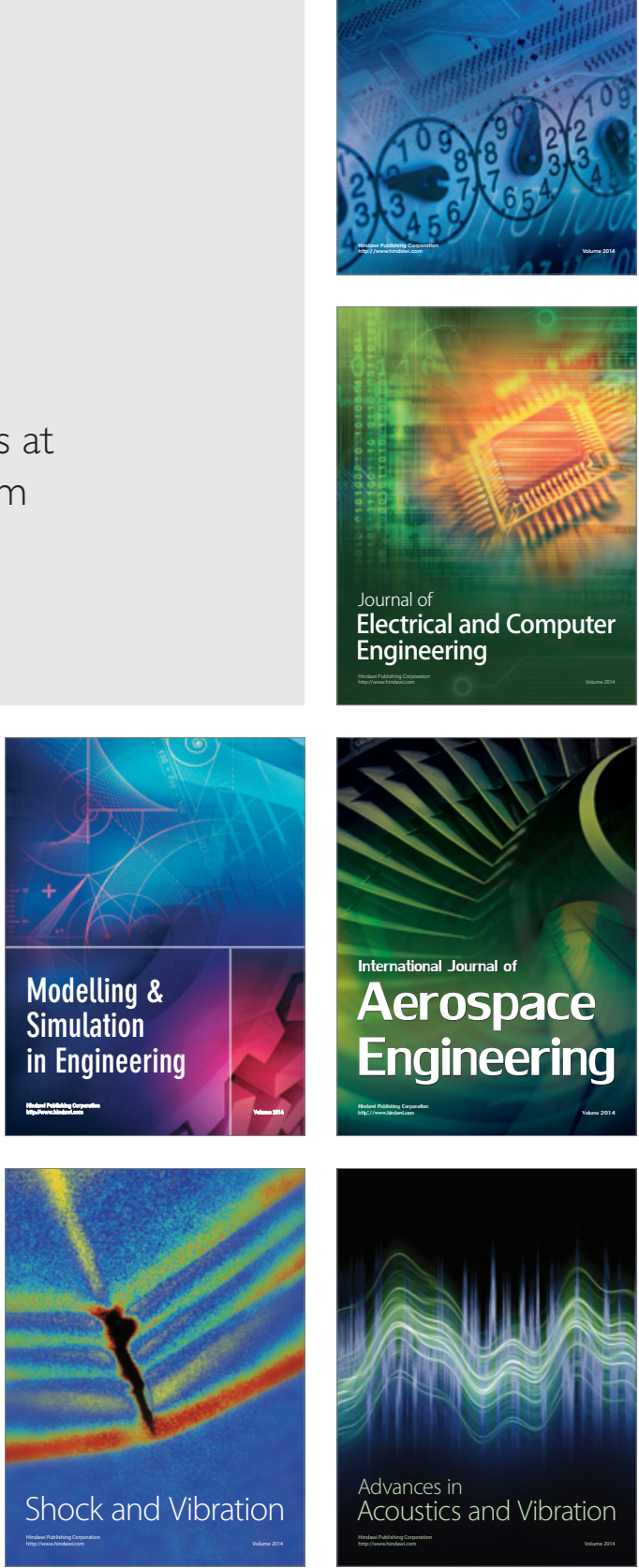